EP-9

\title{
Post-resection prognosis of combined hepatocellular carcinoma-cholangiocarcinoma according to the 2019 WHO classification
}

\author{
Shin HWANG*, Minjae KIM, Chul-Soo AHN, Ki-Hun KIM, Deok-Bog MOON, Gi-Won SONG, Dong-Hwan JUNG \\ Department of Surgery, Asan Medical Center, University of Ulsan College of Medicine, Seoul, Korea
}

Introduction: Combined hepatocellular carcinoma and cholangiocarcinoma (cHCC-CCA) has wide histologic diversity. This study investigated the prognostic impacts of cHCC-CCA histology according to the 2019 World Health Organization (WHO) classification. Methods: This retrospective observational study included 153 patients who underwent surgical resection for cHCC-CCA at Asan Medical Center between August 2012 and July 2019.

Results: During the study period, 153 patients, 112 (73.2\%) men and 41 (26.8\%) women with a mean age of $56.4 \pm 10.8$ years, underwent R0 resection for cHCC-CCA. Mean tumor diameter was $4.2 \pm 2.6 \mathrm{~cm}$, and 147 (96.1\%) patients had solitary tumors. According to 2019 World Health Organization (WHO) classification, 111 (72.5\%) patients had cHCC-CCA alone, and 29 of them (26.1\%) showed stem cell features. cHCC-CCA-intermediate cell carcinoma and cHCC-CCA-cholangiolocellular carcinoma were identified in 27 (17.6\%) and 15 (9.8\%), respectively. The 1-, 3-, and 5-year tumor recurrence and patient survival rates were $31.8 \%$ and $92.1 \%, 49.8 \%$ and $70.9 \%$, and $59.0 \%$ and $61.7 \%$, respectively. Univariate analyses revealed that significant prognostic factors were tumor size $>5 \mathrm{~cm}$, microscopic and macroscopic vascular invasion, lymph node metastasis, 8th American Joint Committee on Cancer (AJCC) tumor stage, and status of stem cell feature. Multivariate analysis revealed 8th AJCC tumor stage and status of stem cell features as independent prognostic factors. The 2019 WHO classification was not associated with post-resection prognosis.

Conclusions: The 2019 WHO classification was not associated with post-resection prognosis, thus was considered as a simple histologic classification. We suggest that stem cell features should be included as an essential component of the pathology report for $\mathrm{cH}$ CC-CCA. 